\title{
A Modified Signal Feed-Through Pulsed Flip- Flop for Low Power Applications
}

\author{
Ehsan Panahifar and Alireza Hassanzadeh
}

\begin{abstract}
In this paper a modified signal feed-through pulsed flip-flop has been presented for low power applications. Signal feed-through flip-flop uses a pass transistor to feed input data directly to the output. Feed through transistor and feedback signals have been modified for delay, static and dynamic power reduction. HSPICE simulation shows $22 \%$ reduction in leakage power and $8 \%$ of dynamic power. Delay has been reduced by $14 \%$ using TSMC 90nm technology parameters. The proposed pulsed flip-flop has the lowest PDP (Power Delay Product) among other pulsed flip-flops discussed.
\end{abstract}

Keywords-low power, pulsed Flip-flop, Delay, leakage power, dynamic power

\section{INTRODUCTION}

$\mathbf{F}$ LIP-FLOPS are basic building blocks of memories and shift registers. Pipelining technique is widely used in recent digital system design that uses flip-flops. It is estimated that more than $50 \%$ of the total circuit power is consumed in clock and storage elements of an integrated circuit [1]. Therefore, power dissipation of flip-flop is very important. Conventional master-slave flip flops do not satisfy timing requirements of high performance systems. Many dynamic schemes such as hybrid latch flip-flop (HLFF) [2], semidynamic flip-flop (SDFF) [3] and sense amplifier-based flipflop (SAFF) [4] [5] have been reported to improve the speed and delay. The major disadvantage of these flip-flops is power consumption, which has been increased due to their dynamic operation and are not useful in low power applications.

Pulsed flip-flops are good replacement for traditional flipflops in high speed circuits [2] [6] [7] [8]. Besides to the speed advantage, their circuit simplicity is also beneficial for lowering the power consumption of the clock tree system. The circuit of a P-FF is simplified since only one latch, as opposed to two latches, are used in conventional master-slave configuration. Since pulsed flip-flops are using only one latch, power dissipation in clock circuit will be reduced too. Pulsed Flip-Flops (P-FF) include a pulse generator and a latch to store data. If the pulse width is too small, latch acts as an edge triggered flip-flop. P-FFs provide time borrowing across clock cycle boundaries and feature a zero or even negative setup time. The pulse generating circuit should be insensitive to process variations [7]. P-FFs are divided into two categories of implicit and explicit, based on pulse generation method [9]. In implicit P-FF, pulse generating circuit is inherent to the circuit and no explicit pulse is generated. Pulse generation and latch circuits are separate in explicit P-FFs. Implicit P-FFs consume

Authors are with ECE Dept., Shahid Beheshti University, Tehran, Iran (e-mail: ehsan.panahy@gmail.com, a_hassanzadeh@sbu.ac.ir). less power than explicit P-FFs, but suffer from slow discharge path. The situation becomes worse when low-power techniques such as conditional capture [10], conditional precharge [11], conditional discharge [12] or conditional data mapping [13] are used. Pulse generation logic transistors are often large to ensure that the generated pulses are sufficiently wide to trigger data latch. Explicit type P-FF designs face a similar pulse width issue. The problem is further complicated in the presence of a large capacitive loads when one pulse generator is shared among several latches [14]. Some implicit P-FFs such as Implicit Data-Close to Output (ip-DCO) [9], Single-ended Conditional Capturing Energy Recovery (SCCER) [6] and Conditional Pulse-Enhancement Scheme $[14]$ have been reported in recent years.

Explicit P-FFs are faster than implicit ones and consume more power, because of separate pulse and latch circuits. If the pulse generating circuit is shared between many P-FFs, power dissipation and circuit complexity is effectively reduced [9] [10] [15]. In this paper a modified P-FF based on the signal feed-through structure [15] has been presented that reduces delay and power dissipation.

This paper has been arranged as follows. Section II reviews the conventional explicit P-FF advantages and disadvantages, then the modified P-FF based on Signal feed-through scheme is introduced. Section III shows simulation results and compares performance of Pulse-triggered flip-flops. Concluding remarks are at the end.

\section{MODIFIED P-FF BASED ON DIRECT SIGNAL FEED- THROUGH}

In this section traditional explicit P-FFs and signal feedthrough P-FFs are reviewed. The principle of operation of each flip-flop is explained as follows.

\section{A- Pulse Generation}

For better understanding of the pulse generation circuits, some pulse generators for P-FFs are discussed in this section. Figure 1-(a) shows a simple pulse generator called clock chopper [16]. This structure consists of a series of inverters that produce an inverted delayed version of pulse clock (CLK signal). The original signal and the delayed signal are passed to a NAND Gate. The width of the pulse is adjustable by setting the delay of the inverters and transistor sizes. The Naffziger's pulsed latch that was used on Itanium 2 processor, is shown in figure 1-(b) [8]. It uses a weak inverter to produce a narrow pulse with a nominal width of about one-sixth of the clock cycle (125 ps for $1.2 \mathrm{GHz}$ clock). Figure 1-(c) shows another pulse generator used on an NEC RISC processor [17]. The generator has a built-in dynamic transmission gate latch to prevent the enable signal glitch during the pulse period [18]. 

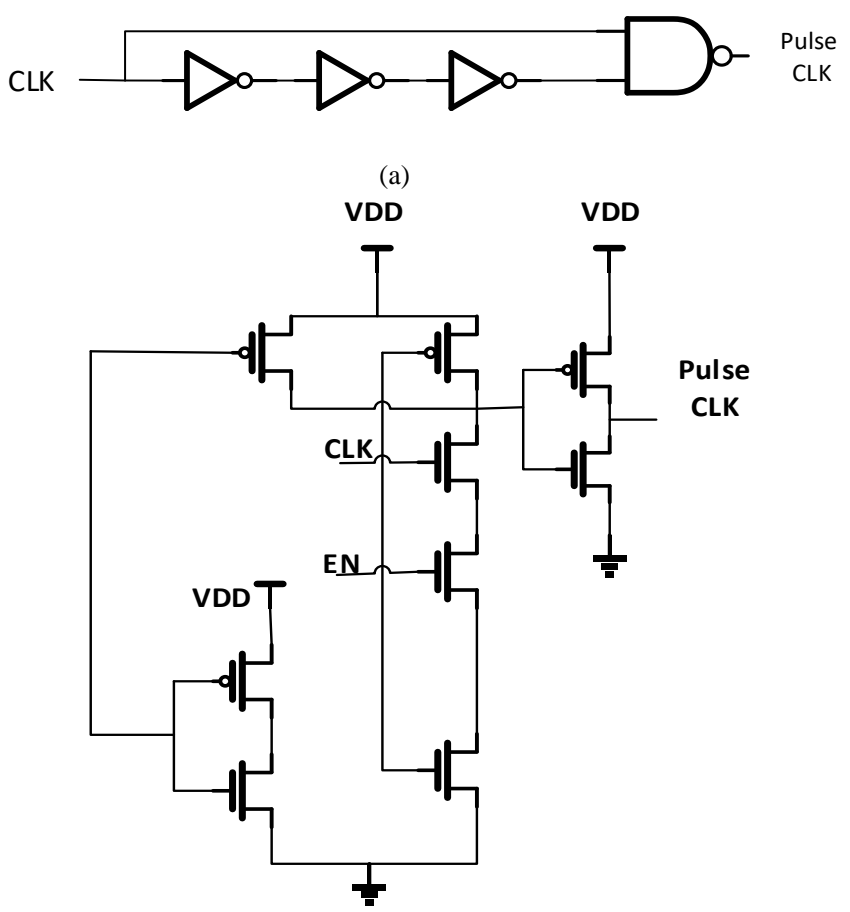

(b)

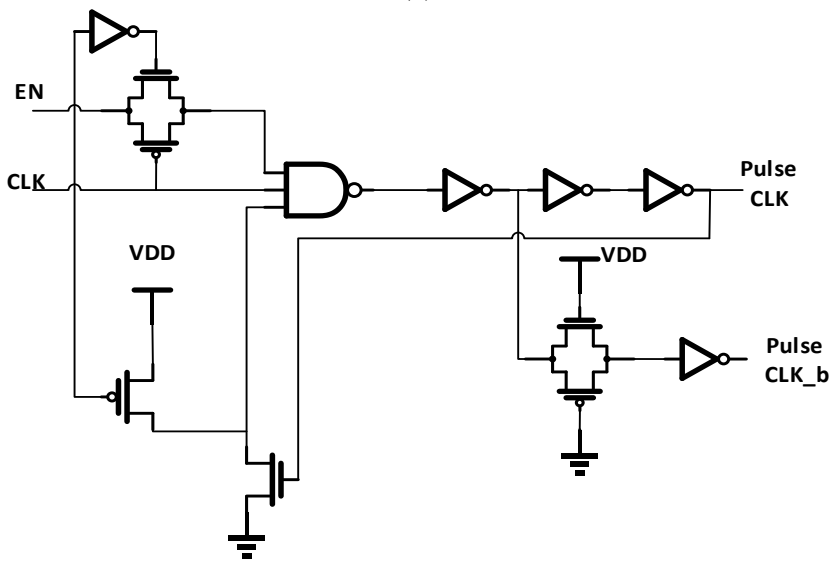

(c)

Fig.1. Pulse generator circuits (a) clock chopper [16] (c) NEC RISC processor [17]

(b) Naffziger [8]

Chopper pulse generator has been used for all P-FF simulations in this paper because of its simple structure. Static CMOS inverters have been used for implementation of delay elements.

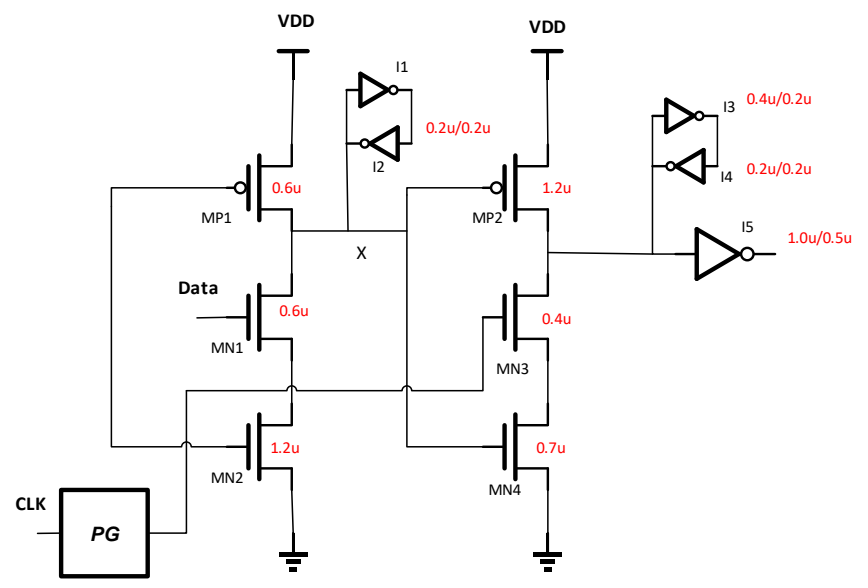

(a)

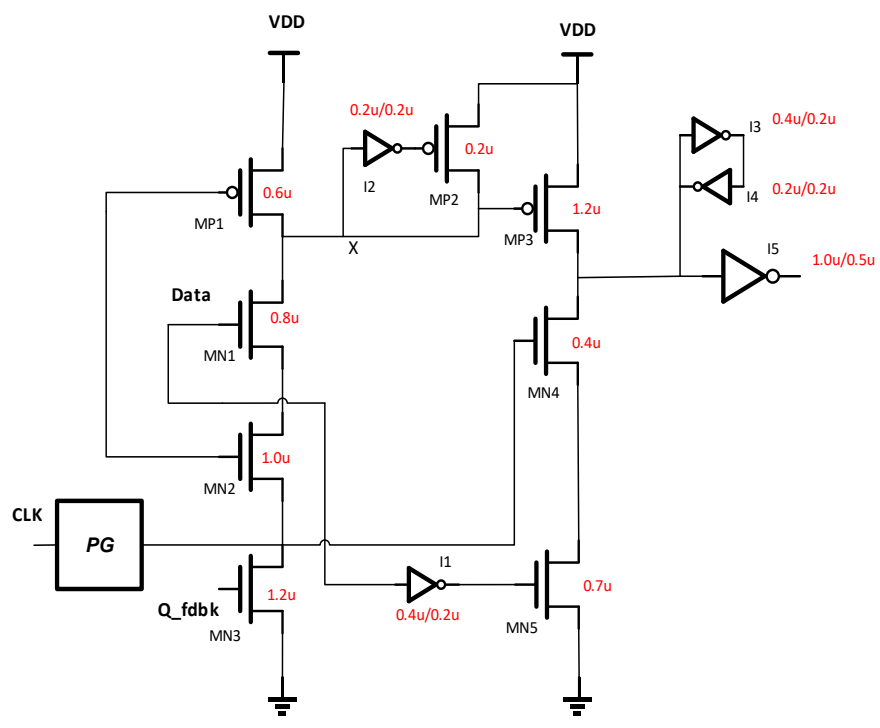

(b)

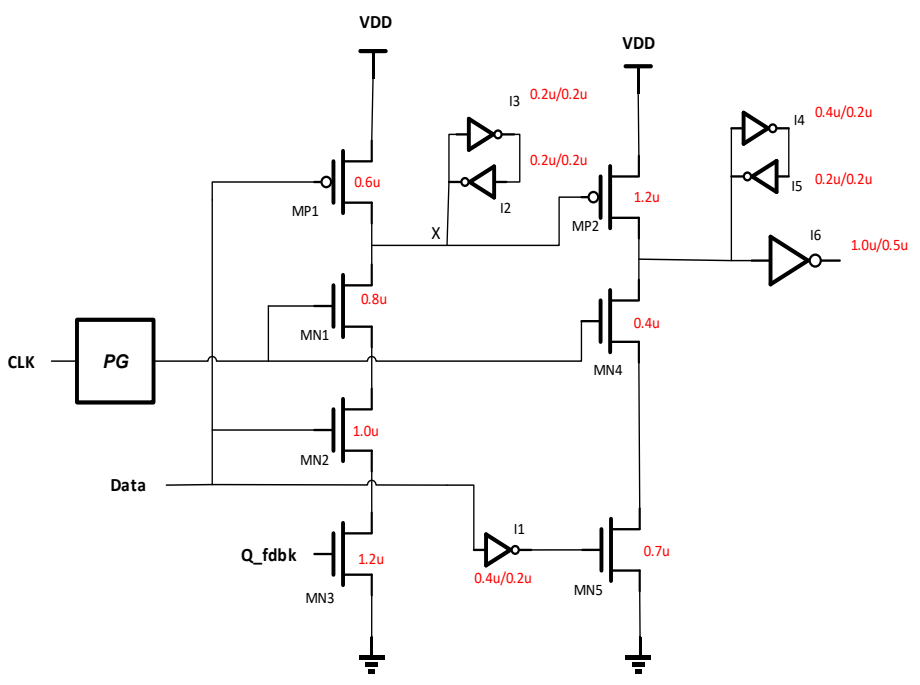

(c)

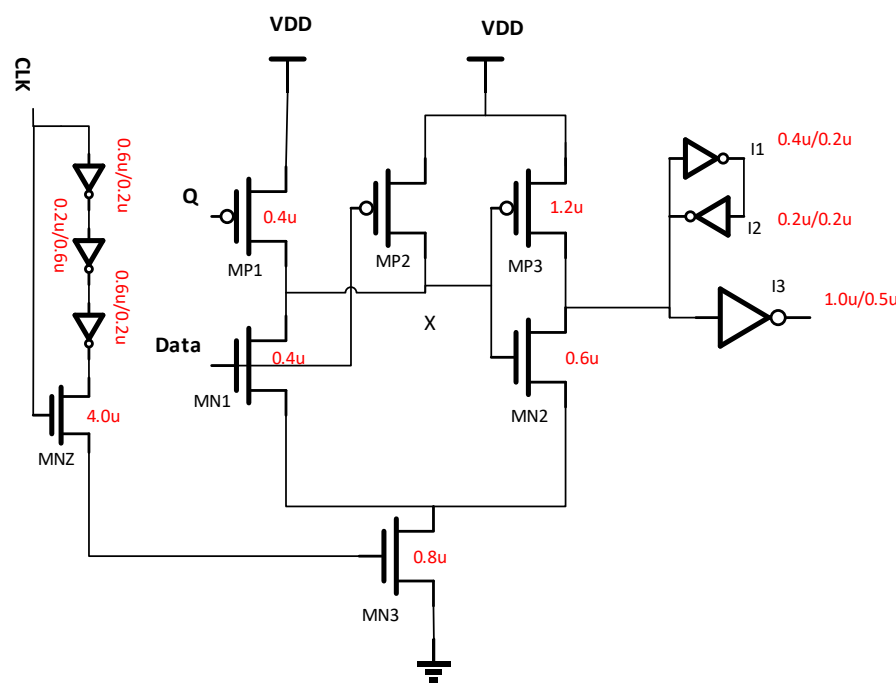

(d)

Fig.2. Conventional P-FF designs: (a) ep-DCO [9] (b) CDFF [12] (c) Static CDFF [19] (d) MHLFF [20] 


\section{$B$ - Traditional Explicit $P-F F$}

Figure 2-(a) shows an explicit P-FF named Explicit DataClose to Output (ep-DCO) [9] that is used as a reference for later comparison. The P-FF uses NAND gates and some inverters to generate a pulse and uses a latch with True-SinglePhase-Clock (TSPC). I3 and I4 inverters are used for data latch and I1 and I2 are used to maintain middle node voltage levels. The clock pulse is generated using three inverters. Point $\mathrm{X}$ is discharged in every rising edge of clock even with " 1 " output state. This produces large switching power dissipation and is a disadvantage of this method. To eliminate this problem many methods have been used such as conditional discharge or CDFF [10]. Figure 2-b shows a CDFF circuit [12]. An NMOS transistor MN3 that is controlled by Q_fdbk signal guarantees that no discharge will happen when output is in "1" state. Also charge keeper for node $\mathrm{X}$ is a pull up PMOS transistor and a simplified inverter. Figure 2-c is a Static Conditional Discharge Flip-Flop (SCDFF) that uses conditional discharge [19]. This FF uses a static latch structure compared to previous one. This FF has more delay of D-to-Q compared to CDFF. Both methods suffer from long discharge path including MN1MN3. For delay reduction a strong pull down circuit is required. A combined latch and FF circuit, Modified Hybrid Latch Flip-Flop or MHLFF, in figure 2-d uses a static latch [20]. In [20] charge keeper at node $X$ has been omitted. A weak pull-up transistor MP1 that is controlled by voltage at Q, keeps X node level when Q is " 0 ". This circuit has two problems, first since node $\mathrm{X}$ is not pre-discharged, long delay is expected for " 0 " to " 1 " transition. This delay is increased because of smaller clock level (reduced by $V_{\text {th }}$ drop). Second, node $\mathrm{X}$ is floating in some cases, which causes larger DC power dissipation.

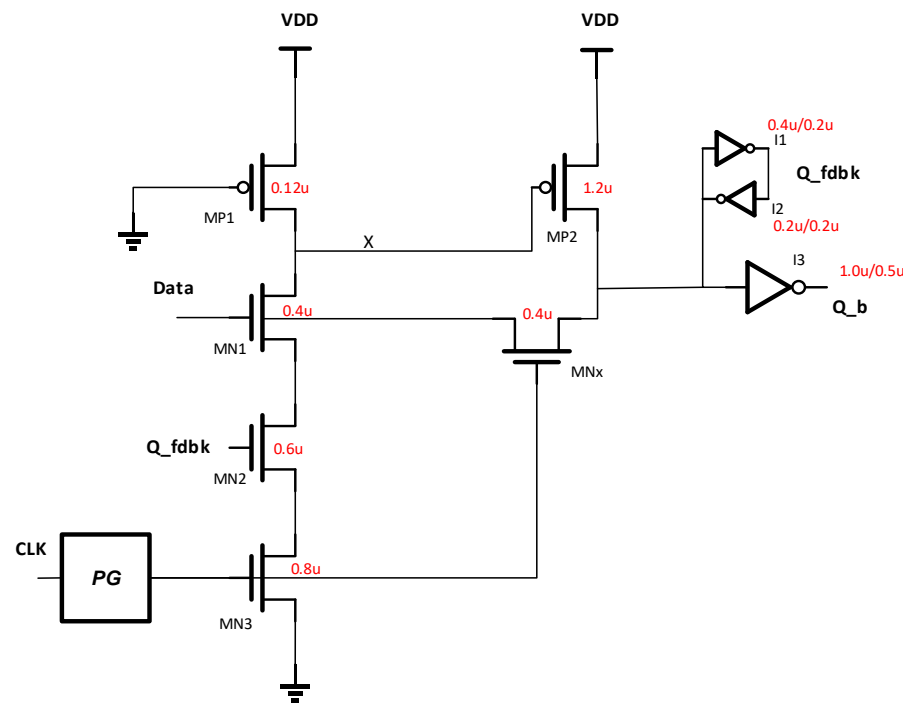

Fig. 3. Pulse-triggered flip-flop design based on a signal feed-through scheme

\section{C- P-FF based on input signal feed-through}

Figure 3 shows a P-FF with signal feed-through structure [15]. This circuit uses a static latch and conditional discharge method. MP1, a weak pseudo-PMOS, is used to keep voltage level at node $\mathrm{X}$. This circuit uses a pass transistor MNX that is controlled by clock pulse and drive output node directly using input data named as signal feed-through. Besides to $\mathrm{MNx}$,
MP2 provides a second path from input to output Q. The pull down network of the latch has been omitted and MNx provides the discharge path. Therefore, $\mathrm{MNx}$ transistor plays the main role for both " 0 " to " 1 " and " 1 " to " 0 " transitions. Comparing to ep-DCO, CDFF and SCDFF, this P-FF has better and balanced delay. However, this circuit has higher leakage power.

The principle of operation of signal feed-through circuit is as follows. If input pulse is applied when there is no input change, Q remains the same. Input data and Q_fdbk are complements. Therefore, pull-down path from $\mathrm{X}$ will be off. When there is input change from " 0 " to " 1 ", node $X$ is discharged and MP2 is turned ON and charges $\mathrm{Q}$ to $\mathrm{V}_{\mathrm{dd}}$ supply. This is the worst time for the FF that the discharge path is connected in such a short pulse time. In this case signal feedthrough, connects input to node $\mathrm{Q}$ using $\mathrm{MNx}$ pass transistor and delay is reduced. When a " 1 " to " 0 " input transition happens, $\mathrm{MNx}$ will be turned $\mathrm{ON}$ by clock pulse and $\mathrm{Q}$ is discharged through $\mathrm{MNx}$ and input stage.

\section{D- The proposed signal feed-through P-FF}

To improve performance of a signal feed-through P-FF, its operation has been characterized. Figure 3 shows percentage of leakage power dissipation of each transistor in the signal feedthrough P-FF circuit.

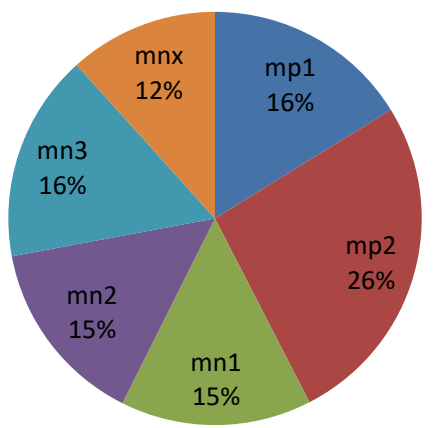

Fig. 4. Leakage power share of each transistor in signal feed-through P-FF

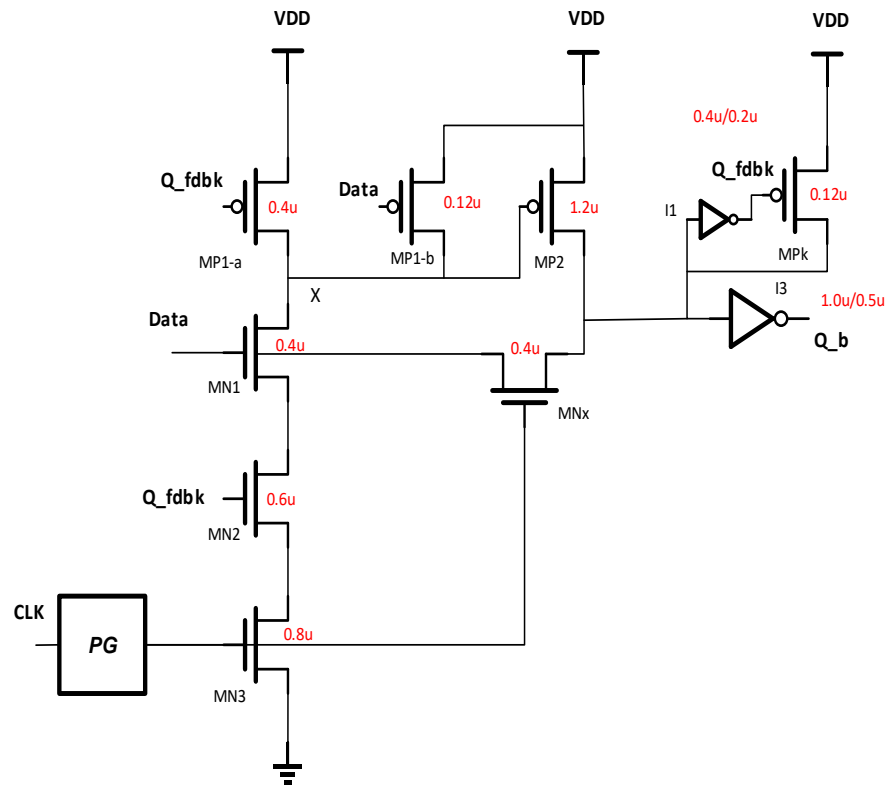

Fig. 5. Modified P-FF based on signal feed-through scheme 
As it can be seen from figure 4, PMOS transistors have higher leakage power share. Therefore, by reducing leakage in MP1, circuit leakage power can be reduced. MP1 is always $\mathrm{ON}$, therefore consumes power in idle state.

MP1 transistor gate is connected to ground. Therefore, it serves as charging and keeping charge at node X. Since MP1 transistor is always ON, it consumes static power. MP1 is a weak pull-up and causes long delay in the circuit too.

To improve performance of the circuit, MP1 has been divided into two transistors that each transistor does the two functions separately.

Transistor MP1-a gate is connected to Q_fdbk and is used for charging node $\mathrm{X}$ when $\mathrm{Q}$ is " 1 ". When output is " 0 ", $\mathrm{Q}$ fdbk is " 1 " and MP1-a will be OFF, this causes less delay because shorter path is created between output and node X.

Another transistor MP1-b with gate connected to Data signal has been added to keep charge at node " $X$ ". When Data is " 0 " node X will be pulled-up by this transistor. Therefore, the always ON PMOS transistor has been omitted and so its static power dissipation.

Instead of two inverters at the output node to keep output charge, MPk, PMOS transistor and an inverter has been used to reduce output delay.

\section{Simulation RESUlts}

For performance evaluation of the proposed circuit HSPICE and TSMC CMOS 90nm technology parameters have been used. For fair comparison all circuits shown in figures 2 and 3 have been simulated. The pulse generation circuit transistors have been sized to produce 120ps pulse. A 20fF load capacitor for the P-FF and a $3 \mathrm{fF}$ capacitor at the clock buffer output have been used [14]. Input data and clock pulse are buffered too. Clock frequency is $500 \mathrm{MHz}$ and $1 \mathrm{~V}$ power supply has been used for simulation. Power has been analyzed for six different data switching activity.

\section{A. Power Consumption Analysis}

Table I summarizes some of the specifications of the P-FFs. As it has been shown in Table I, the proposed circuit has lowest dynamic power consumption comparing to other circuits. Table II shows leakage power dissipation of all circuits. The proposed circuit has superior leakage power dissipation compared to other circuit. Lower leakage power consumption is due to the fact that MP1-a and MP1-b are not always ON. The leakage current of these transistors have been reduced.

Table I

FEATURe COMPARISON OF VARIOUS P-FF DESIGNS

\begin{tabular}{|c|c|c|c|c|c|c|}
\hline FF Design & ep-DCO & CDFF & SCDFF & MHLFF & $\begin{array}{c}\text { Signal feed } \\
\text { through }\end{array}$ & $\begin{array}{c}\text { Modified Signal } \\
\text { feed through }\end{array}$ \\
\hline Number of transistors & 28 & 30 & 31 & 19 & 24 & 24 \\
\hline Setup time (ps) & -73 & -75 & -112 & 1.5 & -82 & -83 \\
\hline Minimum D-to_Q (ps) & 118 & 121 & 138.02 & 116.26 & 107 & 92 \\
\hline Average Power(100\% activity) $\mu \mathrm{W}$ & 30.47 & 29.82 & 35.16 & 26.21 & 26.09 & 24.38 \\
\hline Average Power50\% activity) $\mu \mathrm{W}$ & 24.49 & 23.87 & 26.33 & 23.61 & 21.78 & 20.02 \\
\hline Average Power(25\% activity) $\mu \mathrm{W}$ & 20.79 & 17.93 & 18.4 & 18.16 & 16.56 & 15.3 \\
\hline Average Power(12.5\% activity) $\mu \mathrm{W}$ & 19.99 & 15.36 & 15.28 & 15.94 & 14.43 & 13.31 \\
\hline Average Power(0\% all-1) $\mu \mathrm{W}$ & 20.28 & 12.37 & 12.36 & 13.67 & 12.19 & 11.16 \\
\hline Average Power(0\% all-0) $\mu \mathrm{W}$ & 12.073 & 11.97 & 11.46 & 12.52 & 11.16 & 12.03 \\
\hline Optimal PDP(25\% activity) fJ & 2.18 & 1.97 & 2.45 & 2.11 & 1.67 & 1.41 \\
\hline
\end{tabular}

Table II

LeAKage CuRrent Comparison In StANDBy Mode (NW)

\begin{tabular}{|c|c|c|c|c|c|}
\hline FF Design & ep-DCO & CDFF & SCDFF & $\begin{array}{c}\text { Signal feed } \\
\text { through }\end{array}$ & Modified \\
\hline (CLK, Data $)=(0,0)$ & 51.48 & 53.53 & 58.97 & 53.09 & 47.07 \\
\hline (CLK, Data) $=(0,1)$ & 57.94 & 51.51 & 52.02 & 62.60 & 48.30 \\
\hline (CLK, Data) $=(1,0)$ & 59.87 & 59.56 & 65.31 & 54.32 & 42.80 \\
\hline (CLK, Data) $=(1,1)$ & 66.34 & 67.96 & 74.66 & 63.83 & 44.03 \\
\hline Average & 58.93 & 58.14 & 62.74 & 58.46 & 45.55 \\
\hline
\end{tabular}




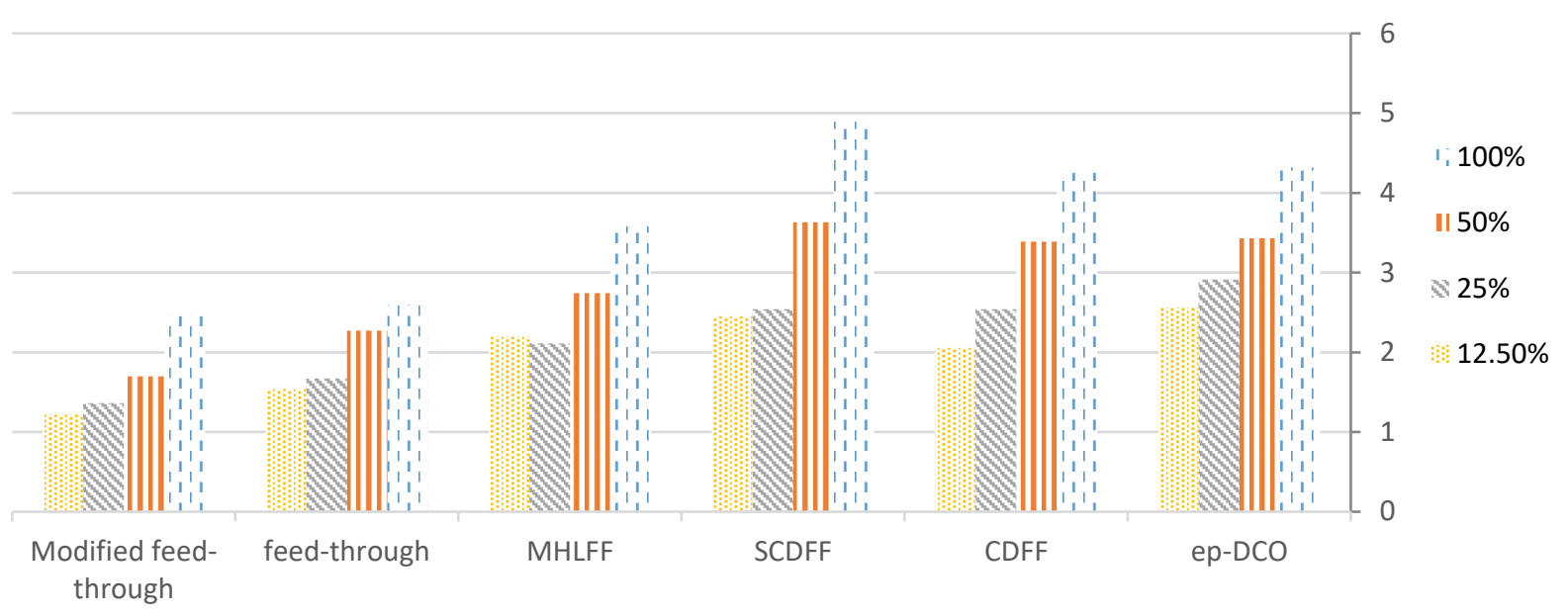

(a)

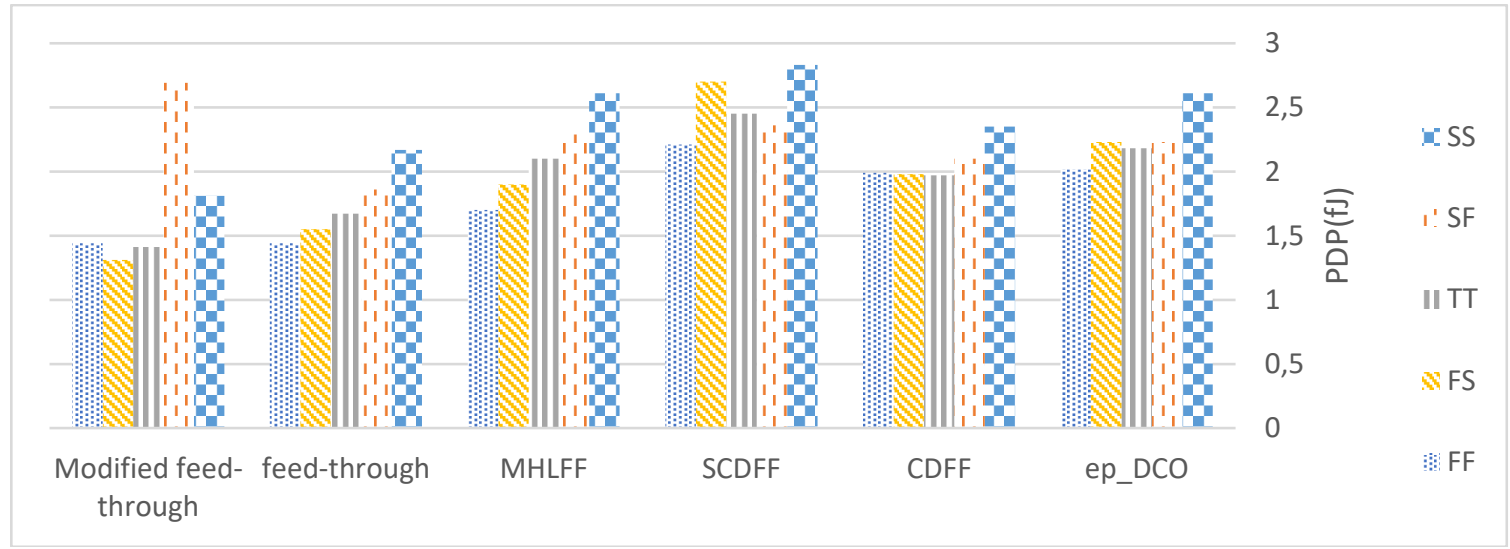

(b)

Fig. 6. PDP performances: (a) Different data switching activity, (b) Different processor corners at $50 \%$ data switching activity.

\section{Simulation Results}

For performance evaluation of the proposed circuit HSPICE and TSMC CMOS 90nm technology parameters have been used. For fair comparison all circuits shown in figures 2 and 3 have been simulated. The pulse generation circuit transistors have been sized to produce 120ps pulse. A 20fF load capacitor for the P-FF and a $3 \mathrm{fF}$ capacitor at the clock buffer output have been used [14]. Input data and clock pulse are buffered too. Clock frequency is $500 \mathrm{MHz}$ and $1 \mathrm{~V}$ power supply has been used for simulation. Power has been analyzed for six different data switching activity.

\section{A. Power Consumption Analysis}

Table I summarizes some of the specifications of the P-FFs. As it has been shown in Table I, the proposed circuit has lowest dynamic power consumption comparing to other circuits. Table II shows leakage power dissipation of all circuits. The proposed circuit has superior leakage power dissipation compared to other circuit. Lower leakage power consumption is due to the fact that MP1-a and MP1-b are not always ON. The leakage current of these transistors have been reduced.

\section{B. Timing Analysis}

As it has been shown in Table I, the minimum D-to-Q delay of the proposed circuit is the lowest among other designs. The power delay product PDP has been improved too and is the lowest for P-FFs. Figure 6-a shows the PDP for different data activities. To consider process variation of PDP for the proposed circuit, simulation has been performed for corner cases of $\left(\mathrm{SS}=0.8 \mathrm{~V} / 125^{\circ} \mathrm{C}, \mathrm{TT}=1 \mathrm{~V} / 25^{\circ} \mathrm{C}, \mathrm{FF}=1.2 \mathrm{~V} /-40^{\circ} \mathrm{C}\right.$, $\mathrm{SF}=1 \mathrm{~V} / 25^{\circ} \mathrm{C}, \mathrm{FS}=1 \mathrm{~V} / 25^{\circ} \mathrm{C}$ ) and the results are shown in figure 6-b. The setup time has been adjusted for best PDP results. The proposed P-FF, when NMOS transistors are faster than PMOS transistors, has the lowest delay and when PMOS transistors are faster, has the highest delay among other methods. The reason is that charging of node $\mathrm{X}$ is dependent on " 0 " to " 1 " transition of Q_fdbk node. This transition is also dependent on discharge time of node $\mathrm{X}$ through NMOS transistors. As a result, when NMOS transistors are faster than PMOS transistors the cycle is executed faster. The proposed circuit has superior performance on other designs except for SF corner case. For TT or typical case, that PMOS transistors are slower, the circuit has acceptable performance 


\section{CONCLUSION}

In this paper a modified signal feed-through P-FF has been proposed. The important modification is to remove always on PMOS transistor and replace with two transistors to separate charging and charge keeping functions. Because of this modification, the static and dynamic power of the P-FF has been reduced by 8 and 22 percent respectively. PDP of the proposed FF is the lowest among other methods. Since large numbers of flip-flops are used in recent digital ICs, the proposed flip-flop modification can improve power dissipation of ICs significantly.

\section{REFERENCES}

[1] H. Kawaguchi and T. Sakurai, "A reduced clock-swing flip-flop (RCSFF) for $63 \%$ power reduction," IEEE J. Solid-State Circuits, vol. 33, pp. 807811, May 1998.

[2] R. Burd, U. Salim, F.Weber, L. DiGregorio, and D. Draper H. Partovi, "Flow-through latch and edge-triggered flip-flop hybrid elements," in IEEE Tech. Dig. ISSCC, pp. 138-139, 1996.

[3] F Klass, "Semi-dynamic and dynamic flip-flops with embedded logic," in Symp. on VLSI Circuits, Dig. of Tech. Papers, pp. 108-109, June 1998.

[4] B. Nikolic et, "Sense amplifier-based flip-flop," Int. Solid-State Circuits Conf., Dig. of Tech, pp. 282-283, Feb. 1999.

[5] M. Matsui et al., "A $200 \mathrm{MHz} 13 \mathrm{~mm}$ 2-D DCT macrocell using sense amplifying pipeline flip-flop scheme," IEEE J. Solid-State Circuits, vol. 29, pp. 1482-1490, 1994.

[6] D. De Caro, E. Napoli, and N. Petra A. G. M. Strollo, "A novel high speed sense-amplifier-based flip-flop," IEEE Trans. Very Large Scale Integr. (VLSI) Syst., vol. 13, pp. pp. 1266-1274, Nov. 2005.

[7] C. Amir, A. Das, K. Aingaran, C. Truong, R.Wang, A. Mehta, R. Heald, and G.Yee F. Klass, "A new family of semi-dynamic and dynamic flip flops with embedded logic for high-performance processors," IEEE J. Solid-State Circuits, vol. 34, pp. 712-716, May 1999.

[8] G. Colon-Bonet, T. Fischer, R. Riedlinger, T. J. Sullivan, and T. Grutkowski S. D. Naffziger, "The implementation of the Itanium 2 microprocessor," IEEE J. Solid-State Circuits, vol. 37, pp. 1448-1460, Nov. 2002.
[9] S. Narendra, Z. Chen, S. Borkar, M. Sachdev, and V. De J. Tschanz, "Comparative delay and energy of single edge-triggered and dual edge triggered pulsed flip-flops for high-performance microprocessors," in Proc. ISPLED, pp. 207-212, 2001.

[10] S. Kim, and Y. Jun B. Kong, "Reduction, Conditional-capture flip-flop for statistical power," IEEE J. Solid-State Circuits, vol. 36, pp. 1263 1271, Aug. 2001.

[11] M. Aleksic, and V. G. Oklobdzija N. Nedovic, "Conditional precharge techniques for power-efficient dual-edge clocking," n Proc Int. Symp.Low-Power Electron. Design, Monterey, pp. 56-59, 2002.

[12] T. Darwish, and M. Bayoumi P. Zhao, "High-performance and low power conditional discharge flip-flop," IEEE Trans. Very Large Scale Integr. (VLSI) Systems, vol. 12, pp. 477-484, May 2004.

[13] M. Hamada, T. Fujita, H. Hara, N. Ikumi, and Y. Oowaki C. K. Teh, "Conditional data mapping flip-flops for low-power and highperformance systems," IEEE Trans. Very Large Scale Integr. (VLSI) Systems, vol. 14, pp. 1379-1383, Dec. 2006.

[14] J.-F. Lin, and M.-H. Sheu Y.-T. Hwang, "Low power pulse triggered flip-flop design with conditional pulse enhancement scheme," IEEE Trans. Very Large Scale Integr. (VLSI) Syst, vol. 20, pp. 361-366, Feb. 2012.

[15] Jin-Fa Lin, "Low-Power Pulse-Triggered Flip-Flop Design Based on a Signal Feed-Through," IEEE Transactions on Very Large Scale Integration (VLSI) Systems, vol. 22, pp. 181 - 185, 2014.

[16] D. Harris, Skew-Tolerant Circuit Design. San Francisco: CA: Morgan Kaufmann, 2001.

[17] S. Kozu et al., "A $100 \mathrm{MHz} 0.4 \mathrm{~W}$ RISC processor with $200 \mathrm{MHz}$ multiply-adder, using pulseregister technique," Proc. IEEE Intl. SolidState Circuits Conf, pp. 140-141, 1996.

[18] David Money Harris Neil H. E. Weste, CMOS VLSI Design:A Circuits and Systems Perspective, 4th ed.: Pearson Education , 2011.

[19] W.-L. Goh, and K.-S. Yeo M.-W. Phyu, "A low-power static dual edge triggered flip-flop using an output-controlled discharge configuration," in Proc. IEEE Int. Symp. Circuits Syst, pp. 2429-2432, May 2005.

[20] A. Khademzadeh, A. Afzali-Kusha, and M. Nourani S. H. Rasouli, "Low power single- and double-edge-triggered flip-flops for high speed applications," IEE Proc. Circuits Devices Syst., vol. 152, pp. 118-122, Apr. 2005. 